\title{
MANEJO Y REHABILITACIÓN DEL MANATÍ AMAZÓNICO (Trichechus inunguis) EN CAUTIVERIO EN EL PERÚ
}

\author{
Carlos Marcial Perea-Sicchar, ${ }^{*}, 1$ Luis Javier Velásquez-Varela, ${ }^{2}$ Juan Sánchez-Babilonia, ${ }^{2}$ Maribel \\ Espinoza-Azan, ${ }^{2}$ Daryl Lee Richardson ${ }^{3}$ y Luis Sigler ${ }^{3}$
}

\author{
${ }^{1}$ Dirección Regional de la Producción del Gobierno Regional de Loreto, calle Ramírez Hurtado 645, Iquitos, Perú. \\ e-mail: maici_perea@yahoo.com \\ ${ }^{2}$ Asociación para la Conservación de la Biodiversidad Amazónica - Dallas World Aquarium Zoo, carretera Iquitos - Nauta \\ $\mathrm{Km}$ 4.5, Iquitos, Perú \\ ${ }^{3}$ The Dallas World Aquarium, 1801 N. Griffin St., Dallas, Texas 75202 USA
}

\begin{abstract}
RESUMEN
La caza furtiva de adultos y la comercialización de crías de manatí amazónico o vaca marina Trichechus inunguis en el Perú, especialmente en la selva baja del departamento de Loreto, ha originado la necesidad de contar con normas técnicas apropiadas para el manejo y rehabilitación de esta especie en condiciones de cautiverio, lo que conllevó a la conformación de la "Fundación Iquitos - Centro de Rescate Amazónico", un esfuerzo interinstitucional que está involucrado en la conservación y protección de mamíferos acuáticos amazónicos. El Centro de Rescate Amazónico, atiende a 13 manatíes y asiste a otros 4 ubicados en otros predios, logrando la rehabilitación de varios de ellos que se encuentran aptos para su liberación; además que ha generado el inicio de investigaciones y el desarrollo de actividades de educación ambiental relacionados a esta especie.
\end{abstract}

Palabras clave: Trichechus inunguis, manatí amazónico, cautiverio, rehabilitación, conservación

\section{MANAGEMENT AND REHABILITATION OF THE AMAZONIAN MANATEE ( Trichechus inunguis) IN CAPTIVITY IN PERU}

\begin{abstract}
The poaching of adults and the commercialization of Amazonian manatee calves Trichechus inunguis or sea cow still occurring in Peru, especially in the lowland of the Loreto department. This situation has created the need of technical and appropriate standards for the management and rehabilitation of this species in captivity. This gap led to the formation of the "Iquitos Foundation Amazon Rescue Center", an interagency effort that is involved in conservation and protection of the Amazonian aquatic mammals. The centre caters 13 manatees and assists 4 located off-site, achieving the rehabilitation of several of them that currently are suitable for release; also, the Amazon Rescue Center has started research activities and development of related environmental education.
\end{abstract}

Keywords: Trichechus inunguis, amazonian manatee, captivity, rehabilitation, conservation 


\section{INTRODUCCIÓN}

Los sirenios son los únicos mamíferos acuáticos herbívoros, presentes únicamente en regiones tropicales, particularmente en ambientes marinos y dulce acuícolas. Este grupo ha enfrentado a lo largo de su historia evolutiva amenazas naturales y más recientemente antrópicas, que han hecho que su supervivencia sea una prioridad para muchos gobiernos e instituciones (Caicedo-Herrera et al, 2005)

A pesar de la legislación peruana, el manatí amazónico es capturado regularmente en lagunas de agua negra y quebradas dentro y en las cercanías de la Reserva Nacional Pacaya Samiria. Gran parte de la explotación del manatí en esta región ocurre conjuntamente con la pesca del paiche, Arapaima gigas. Los manatíes son además cazados de manera oportunista durante las faenas de pesca en otros lugares de la Amazonia peruana (Reeves et al., 1996). Además, la caza furtiva de hembras de vaca marina o manatí amazónico origina el comercio ilegal de crías huérfanas como mascotas los cuales son mantenidos en estanques o para su consumo posterior (Perea et al., 2009)

\section{Aspecto Legal}

En el Perú el Reglamento de Ordenamiento Pesquero de la Amazonía Peruana inicialmente aprobada en el 2001 a través de la Resolución Ministerial No 147-2001-PE del entonces Ministerio de Pesquería, es actualizada en el 2009 mediante el Decreto Supremo № 015-2009-PRODUCE del Ministerio de la Producción, donde se prohíbe la extracción, procesamiento y comercialización de la especie de sirenio Trichechus inunguis (manatí amazónico o vaca marina) y delfines amazónicos con fines de consumo humano y/o para mantenimiento de ejemplares vivos en cautiverio y cualquier otro fin; a menos que provengan de acciones de rescate, decomiso y captura incidental, en cuyo caso pueden mantenerse en cautiverio sólo temporalmente, para su rehabilitación antes de ser devueltos al ambiente natural.

En el 2007 el Decreto Supremo No 016-2007-PRODUCE del Ministerio de la Producción, aprueba el Reglamento de Inspecciones y Sanciones Pesqueras y Acuícolas (RISPAC); donde se incluye sanciones a las personas naturales o jurídicas que atentan contra los mamíferos acuáticos amazónicos.

Por otro lado, el Decreto Supremo No 034-2004-AG del Ministerio de Agricultura, aprueba la categorización de especies amenazadas de fauna silvestre y prohíben su caza, tenencia, transporte o exportación con fines comerciales; donde el manatí es catalogado como en peligro.

Asimismo, la Ordenanza Regional No 018-2008-GRL/CR del Gobierno Regional de Loreto en el año 2008, establece las Normas Técnicas de las Condiciones ambientales y de cuidado para el adecuado mantenimiento y bienestar de mamíferos acuáticos amazónicos (bufeo colorado Inia geoffrensis, bufeo negro Sotalia fluviatilis y manatí amazónico Trichechus inunguis) en cautiverio con fines de rehabilitación. Este dispositivo legal es ampliado y modificado al año siguiente con la Ordenanza Regional No 009-2009-GRL/CR.

En junio de 2010, con el apoyo de ONGs como WWF, WCS, Pro Naturaleza y la Fundación Omacha y con la participación de autoridades locales del departamento de Loreto y nacionales, se lleva a cabo en la ciudad de Iquitos un taller de bases para la construcción de un Plan de Acción en torno a delfines de río y manatí en la Amazonía peruana, la misma que está a la espera de los resultados para que sea incluido dentro de las políticas del Ministerio de la Producción y de los Gobiernos Regionales del ámbito amazónico. 


\section{Manejo y Cautividad}

Los manatíes amazónicos han sido mantenidos satisfactoriamente en cautiverio en el Instituto Nacional de Pesquisas da Amazônia en Manaos, Brasil desde 1974, entre adultos y crías huérfanas. Los ejemplares adultos se adaptaron fácilmente al cautiverio (Rosas, 1994)

El Proyecto Conservaçâo e Manejo do Peixe-boi da Amazônia del Brasil es pionero en la manutención de individuos de manatí amazónico en cautiverio. El proyecto, llevado a cabo por el Laboratório de Mamíferos Aquâticos (LMA/INPA) contempla el estudio simultáneo de diferentes aspectos de la biología de esta especie en cautiverio (salud, nutrición, reproducción y comportamiento). El objetivo del proyecto es establecer un programa piloto de crianza en cautiverio, hacia una mayor producción y manejo adecuados de los stocks existentes para la conservación de la especie. De esta manera, se han realizado estudios sobre la alimentación y nutrición (formulas lácteas para la alimentación de crías huérfanas, caracterización de las heces de crías y adultos, preferencia alimenticia en cautividad), reproducción (extracción de hormonas reproductivas en machos) y estudio de comportamiento en cautiverio (identificación de las características y funciones de las vocalizaciones) (da Silva, 1999)

Estas experiencias de cautividad de $T$. manatus y $T$. inunguis también se ha desarrollado en la república de Colombia, donde reportan estos animales en cautiverio y semicautiverio en las cuencas de los ríos Magdalena, Antro y Sinu, Orinoco y Amazonas; además cuentan con un Programa Nacional de Manejo y Conservación de Manatíes en Colombia (Caicedo-Herrera et al, 2005)

En Perú, a inicios de setiembre de 2007, se implementó un programa para el rescate, rehabilitación y preparación para la liberación de manatíes huérfanos en la Amazonía peruana, complementada con campañas de divulgación dirigidas a comunidades ribereñas, con el propósito de evitar la caza, comercio ilegal de crías y la conservación de su hábitat (Velásquez-Varela et al., 2009).

\section{MATERIAL Y MÉTODOS}

Las aspectos técnicos y legales establecidos para el manejo y rehabilitación de mamíferos acuáticos amazónicos se encuentran contenidos en la Ordenanza Regional No 018-2008-GRL/CR, los mismos que fueron adoptados por la Fundación Iquitos - Centro de Rescate Amazónico, que es administrada por ACOBIA-DWAZOO, único autorizado en el Perú para este propósito. Las instalaciones provisionales se ubican en el Programa de Investigación para el Uso Conservación del Agua y sus Recursos (AQUAREC) del Instituto de Investigaciones de la Amazonía Peruana, en el $\mathrm{Km} \mathrm{4,5}$ de la carretera Iquitos - Nauta, distrito de San Juan Bautista, provincia de Maynas, departamento de Loreto.

\section{Obtención de los ejemplares de manatí amazónico:}

Los manatíes provienen de los decomisos o labores de rescate que realiza la Dirección Regional de la Producción del Gobierno Regional de Loreto, con el apoyo de ACOBIA-DWAZOO los cuales son trasladados a las instalaciones de la Fundación Iquitos - Centro de Rescate Amazónico (FI-CREA) para su rehabilitación. 


\section{Manejo Sanitario}

Para el tratamiento veterinario de los animales, se realizó el manejo sanitario con el uso de diversos fármacos orientado por varios aspectos como son los análisis de laboratorio, el examen de sensibilidad antimicrobiana, los signos clínicos y la edad del animal principalmente. En este sentido, se cuenta con fármacos básicos y específicos para encarar situaciones de emergencia, como para tratar enfermedades de origen infeccioso. La utilización de fármacos con los animales que se encuentran en las pozas de cuarentena y las de rehabilitación son:

- Yodo Povidona: Antiséptico que será usado en forma tópica, en el caso de heridas en superficie corporal.

- Antimicrobianos tropicales: antibióticos que son aplicados en superficie corporal para el tratamiento de heridas externas. Contienen antibiótico, antiinflamatorio y antimicótico (ejemplo: Notil, Iconyl, entre otros)

- Antibióticos orales: utilizado en el caso de trastornos digestivos y pulmonares (ejemplo: Metronidazol, Cefalosporinas, entre otros)

- Antibióticos sistémicos: utilizado en cuadros infecciosos de curso rápido (Ciproflacina, Enrofloxacina, etc.) según la orientación por la prueba de sensibilidad antimicrobiana. Se administra por vía Intramuscular. No se utilizan quinolonas en crías ni juveniles.

- Antiparasitarios y antimicóticos: se utiliza un fármaco específico previo diagnostico de laboratorio.

\section{Registro}

Los manatíes son registrados a través del implante de microchips tipo Angel o AVID (que permite también el registro de temperatura corporal), fotografías de machas y cicatrices, biometría, toma de muestra genética, entre otros.

\section{Infraestructura}

Se utiliza cuatro pozas o estanques de cuarentena para crías huérfanas de manatí amazónico, cada una con las siguientes características:

- Material: noble

- Pared interna: mayólicas

- $\quad$ Dimensiones: longitud = 3,5 m; ancho $=2,00 \mathrm{~m}$; profundidad $=0,85 \mathrm{~m}$

- Área: $7 \mathrm{~m}^{2}$

- Sistema de llenado y vaciado (válvulas): El llenado de las pozas proviene de las pozas de tratamiento de agua, el vaciado es mediante tubos instalados en la parte inferior de las pozas.

También para rehabilitación de manatíes amazónicos juveniles/ sub adultos/ adultos se realiza en una poza, con las siguientes características:

- Material: noble

- Pared interna: cemento pulido

- $\quad$ Dimensiones: longitud $=15,4 \mathrm{~m}$; ancho $=7,30 \mathrm{~m}$; profundidad $=1,2 \mathrm{~m}$

- Área: $112,42 \mathrm{~m}^{2}$

- Decantadores: Esta poza contiene un sistema de decantación y filtración, lo que permite mantener el agua en buenas condiciones por mayor tiempo.

- Sistema de llenado y vaciado (válvulas): Esta poza se llena del agua proveniente de los tanques de tratamiento y de un pozo artesiano de $80 \mathrm{~m}$ de profundidad; para el vaciado se utiliza el tubo de desagüe instalado en la parte inferior del mismo. 
Asimismo, para proceso de readaptación de manatíes amazónicos juveniles/ sub adultos/ adultos se lleva a cabo en un estanque de tierra con las siguientes características:

- Material: arcilloso

- Pared interna: arcilloso

- $\quad$ Dimensiones: longitud $=31 \mathrm{~m}$; ancho $=24 \mathrm{~m}$; profundidad $=0,5-1,8 \mathrm{~m}$

- Área: $744 \mathrm{~m}^{2}$

- Sistema de llenado y vaciado: El agua de proviene de las instalaciones de reproducción inducida del AQUAREC y de vacía mediante un codo móvil.

\section{Tratamiento, desinfección y/o mantenimiento de las pozas:}

Las pozas son limpiadas diariamente con la ayuda de un jamo telescópico y mediante el cambio y/o recirculación y filtrado del agua. Se retira mecánicamente los restos de alimentos y heces. Semanalmente las pozas son desinfectadas con Dodigen ${ }^{\circledR}$ (antibacteriano y antifúngico para piscinas) y se cuenta con tres flotadores que contienen pastillas de cloro, las cuales son cambiadas semanalmente o de acuerdo al desgaste de las mismas. También con la ayuda de escobillas se retiran los detritos y otras impurezas de las paredes y fondo de las pozas.

\section{Sistema de recirculación y filtración del agua}

El agua es extraída del subsuelo mediante una electrobomba sumergible de 2 HP de potencia de un pozo artesiano de $80 \mathrm{~m}$ de profundidad. El agua deriva hacia tres pozas de concreto (longitud: $4 \mathrm{~m}$; ancho: 2,5 m; profundidad: $1,55 \mathrm{~m}$ ) para el tratamiento respectivo. Se utiliza un filtro de alta velocidad de 22" de diámetro con una electrobomba de $1,5 \mathrm{HP}$ de potencia y $18 \mathrm{~m}^{3} / \mathrm{hora}$ de capacidad. La primera poza sirve para almacenamiento, la segunda se agrega aditivos como sulfato de aluminio y cloro, la tercera sirve para la recirculación y filtración a través del filtro de alta velocidad y tres filtros grandes artesanales. En el sistema de decantación de la poza de rehabilitación de manatíes, también cuenta con un filtro de alta velocidad y electrobomba y tres filtros grandes artesanales con características similares, de este modo el agua es recirculado y filtrado nuevamente.

\section{Alimentación}

Todas las crías recuperadas son rehidratadas vía oral con suero fisiológico y dextrosa al 5\%, durante tres a cinco días, luego gradualmente se le agrega leche Milkmatrix ${ }^{\circledR}$ 33/40 para mamíferos. Aproximadamente al mes se alcanza una dilusión de 14 cucharadas de leche por 250 $\mathrm{mL}$ de agua, la cual es ofrecida cada 4 a 6 veces al día, esta fórmula, dependiendo del animal, se le agrega 20 gotas de simeticona con la finalidad de ayudar a la evacuación de gases o el agua es reemplazado por dextrosa al 5\% cuando se observa deshidratación.

Alrededor de los tres meses de rehabilitación de las crías se les empieza a ofrecer alimento sólido, siendo la huama (Pistia stratiotes) la principal fuente de alimento, ocasionalmente se les ofrece banana (Musa paradisiaca) y papaya (Carica papaya). El destete se inicia a los dos años disminuyendo el consumo de leche hasta dos veces al día.

Las crías en este proceso son trasladadas a la poza de rehabilitación, donde permanecen hasta alcanzar los $70 \mathrm{~kg}$ de peso. Luego se trasladan a la poza de readaptación o preliberación. En los estadios juveniles y adultos son alimentados exclusivamente con Pistia stratiotes, el cual es ofrecido ad libitum. 


\section{Recursos Humanos}

La Fundación Iquitos - Centro de Rescate Amazónico está conformado por profesionales capacitados y adiestrados en el manejo y rehabilitación de mamíferos acuáticos amazónicos, así como de profesionales en educación ambiental. Cuenta además con la supervisión de médicos veterinarios locales y del Dallas World Aquarium.

\section{RESULTADOS Y DISCUSIÓN}

\section{Rescate y Rehabilitación}

Durante los años 2007-2010, se logró el rescate de 28 manatíes, de los cuales 17 se encuentran vivos ( 2 crías, 9 juveniles, 6 adultos). De éstos, 10 son hembras y 7 son machos (ver Cuadro $N^{\circ} 1$ )

Cuadro N 1: Manatíes amazónicos cautivos del Departamento de Loreto

\begin{tabular}{|c|c|c|c|c|c|c|c|}
\hline Nombre & Sexo & $\begin{array}{l}\text { Edad } \\
\text { Aprox. }\end{array}$ & $\begin{array}{l}\text { Grupo } \\
\text { Etáreo }\end{array}$ & Procedencia & Origen & $\begin{array}{c}\text { Lugar de } \\
\text { Cautiverio }\end{array}$ & $\begin{array}{l}\text { Tiempo de } \\
\text { Cautividad }\end{array}$ \\
\hline Daniela & $\mathrm{H}$ & $\begin{array}{l}6 \mathrm{a}, 2 \\
\mathrm{~m}\end{array}$ & Adulto & Río Ucayali & Mascota & Granja 4 & $3 a, 2 m$ \\
\hline Marvino & $M$ & $\begin{array}{l}6 a, 2 \\
m\end{array}$ & Adulto & ¿? & Mascota & Pilpintuwasi & $3 a, 2 m$ \\
\hline Sebastián & $M$ & $\begin{array}{l}6 \mathrm{a}, 2 \\
\mathrm{~m}\end{array}$ & Adulto & Río Marañón & Mascota & $\begin{array}{l}\text { Fundo } \\
\text { Arapaima } \\
\text { Gigas }\end{array}$ & $3 a, 2 m$ \\
\hline Juliana & $\mathrm{H}$ & $\begin{array}{l}7 \mathrm{a}, 2 \\
\mathrm{~m}\end{array}$ & Adulto & $\begin{array}{l}\text { Río } \\
\text { Amazonas }\end{array}$ & Mascota & FI-CREA & $4 a, 2 m$ \\
\hline Suramérica & $H$ & $\begin{array}{l}4 a, 2 \\
m\end{array}$ & Adulto & Río Samiria & Mascota & FI-CREA & $3 \mathrm{a}, 8 \mathrm{~m}$ \\
\hline Yanayacu & $H$ & $\begin{array}{l}3 a, 2 \\
m\end{array}$ & Juvenil & $\begin{array}{l}\text { Río } \\
\text { Yanayacu, } \\
\text { afluente Río } \\
\text { Napo } \\
\end{array}$ & Mascota & FI-CREA & $3 \mathrm{a}, 1 \mathrm{~m}$ \\
\hline Yacuruna & $M$ & $\begin{array}{l}3 a, 2 \\
m\end{array}$ & Juvenil & $\begin{array}{l}\text { Río } \\
\text { Chambira, } \\
\text { fluente Río } \\
\text { Marañón }\end{array}$ & Mascota & Granja 4 & $2 a, 11 \mathrm{~m}$ \\
\hline Aántu & $M$ & $\begin{array}{l}3 a, 2 \\
m\end{array}$ & Juvenil & Río Tigre & Mascota & FI-CREA & $2 \mathrm{a}, 6 \mathrm{~m}$ \\
\hline Chamo & $M$ & $\begin{array}{l}2 a, 4 \\
m\end{array}$ & Juvenil & Río Ucayali & Mascota & FI-CREA & $2 a$ \\
\hline Santa Cruz & $M$ & $\begin{array}{l}2 a, 4 \\
m\end{array}$ & Juvenil & Río Marañón & Mascota & FI-CREA & $1 \mathrm{a}, 7 \mathrm{~m}$ \\
\hline $\begin{array}{l}\text { Victoria } \\
\text { Regia }\end{array}$ & $\mathrm{H}$ & $\begin{array}{l}2 a, 6 \\
m\end{array}$ & Juvenil & ¿? & Comercialización & FI-CREA & $1 \mathrm{a}, 6 \mathrm{~m}$ \\
\hline Cochy & $\mathrm{H}$ & $\begin{array}{l}3 a, 9 \\
m\end{array}$ & Juvenil & $\begin{array}{l}\text { Río } \\
\text { Amazonas }\end{array}$ & Mascota & FI-CREA & $1 \mathrm{a}, 9 \mathrm{~m}$ \\
\hline Quino & $M$ & $\begin{array}{l}5 a, 9 \\
m\end{array}$ & Adulto & $\begin{array}{l}\text { Río } \\
\text { Amazonas }\end{array}$ & Mascota & FI-CREA & $11 \mathrm{~m}$ \\
\hline Requena & $\mathrm{H}$ & $3 a$ & Juvenil & $\begin{array}{l}\text { Río Ucayali } \\
\text { (¿?) }\end{array}$ & Mascota & FI-CREA & $2 \mathrm{a}, 7 \mathrm{~m}$ \\
\hline Nautina & $\mathrm{H}$ & $10 \mathrm{~m}$ & Cría & Río Marañón & Mascota & FI-CREA & $8 \mathrm{~m}$ \\
\hline Yuri & $\mathrm{H}$ & $7 \mathrm{~m}$ & Cría & Río Huallaga & Mascota & FI-CREA & $1 \mathrm{~m}$ \\
\hline $\begin{array}{l}\text { Marina } \\
\text { Itaya }\end{array}$ & $\mathrm{H}$ & $3 a$ & Juvenil & $\begin{array}{l}\text { Río } \\
\text { Amazonas }\end{array}$ & Comercialización & FI-CREA & $1 \mathrm{~m}$ \\
\hline
\end{tabular}

Fuente: Dirección Regional de la Producción del Gobierno Regional de Loreto, ACOBIA-DWAZOO. 
Como se aprecia en el cuadro precedente, el principal origen de la tenencia ilegal de estos animales es el uso como mascota. Por otro lado, lugares como La Granja 4, Pilpintuwasi y Fundo Arapaima Gigas tienen en total 4 manatíes adultos que son supervisados por la Dirección Regional de la Producción y monitoreados por ACOBIA-DWAZOO.

Entre las principales causas de mortandad se reportó neumonía crónica y enteritis necrótica amebiana, que afectaba a crías que llegaban con muy bajo peso y con infecciones derivadas por heridas con arpón, machete y con altos niveles de desnutrición y deshidratación.

En cuanto al crecimiento de los animales, podemos colocar como ejemplo a siete ejemplares: Suramérica, Yanayacu, Aántu, Yacuruna, Chamo, Victoria y Santa Cruz, quienes llegaron con un promedio de 20,04 kg de peso y 104,57 cm de longitud; éstos llegaron alcanzar en 308 días de rehabilitación 47,89 kg y 127,47 cm, y a los 1029 días (Suramérica, Yanayacu, Aántu) 93,00 kg y $161,23 \mathrm{~cm}$ respectivamente. El detalle del crecimiento podemos observarlo en la siguiente figura:

Figura 1: Crecimiento en peso $(A)$ y longitud $(B)$ de 7 ejemplares de $T$. inunguis en rehabilitación en la FI-CREA.

Fuente: ACOBIA-DWAZOO

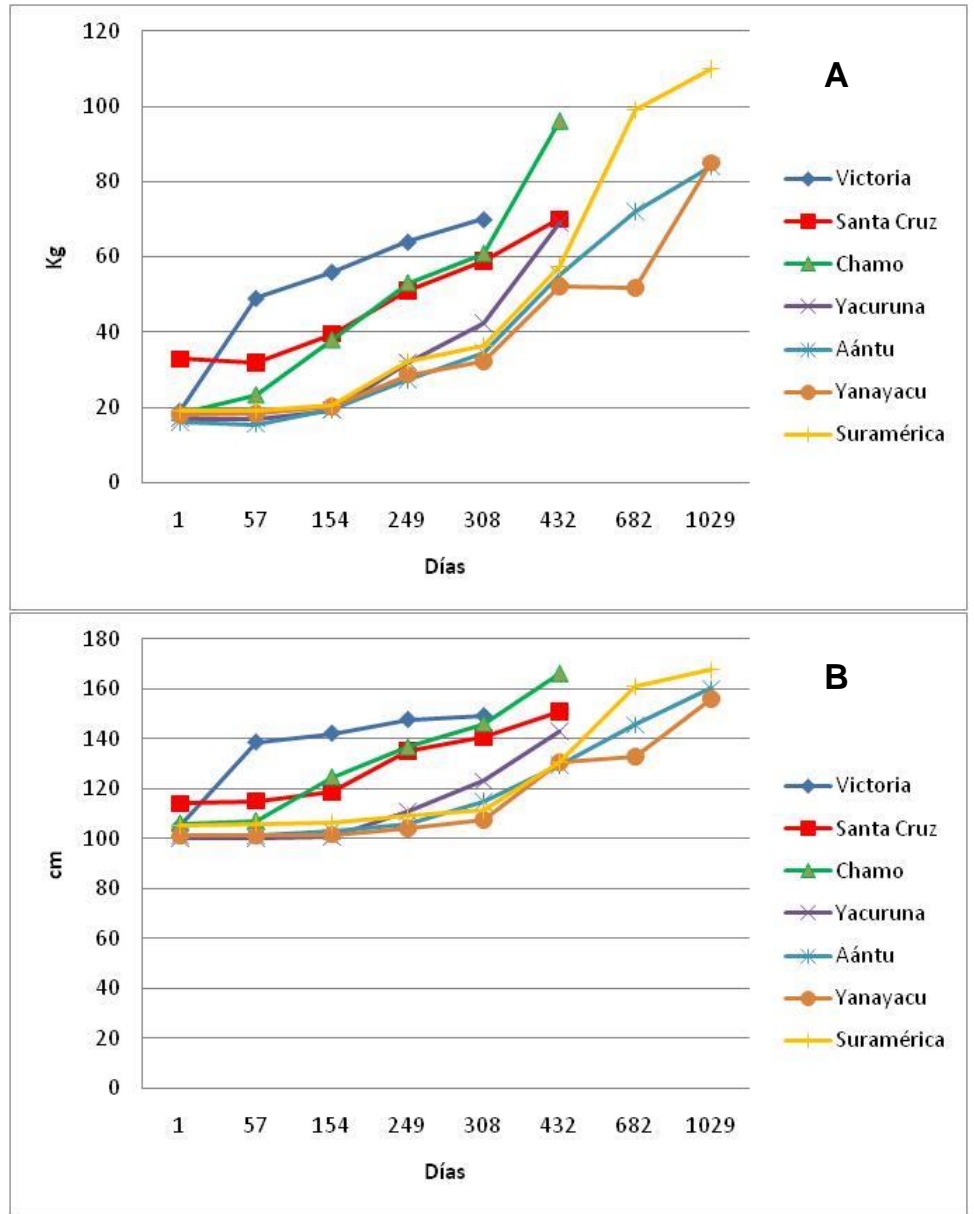

\section{Acciones de liberación}

Conforme al protocolo de liberación establecido en la O.R. № 018-2008-GRL-CR, en junio de 2011 se efectuó la liberación de 5 ejemplares de manatí amazónico rehabilitados en la Cocha El Dorado, ubicado en la cuenca Yanayacu Pucate de la Reserva Nacional Pacaya Samiria en el departamento de Loreto. Para ello se les colocó un radiotransmisor VHF en el pedúnculo caudal y se utilizó antenas de recepción tipo Yagui, para permitir el seguimiento y monitoreo de los animales. 


\section{Educación ambiental}

Como parte de las labores de sensibilización y difusión de normas legales, la FI-CREA en el 2008 inicia una ardua labor de educación ambiental, a través de charlas, talleres, exhibición de títeres, visitas al Centro, entrevistas televisivas, radiales y escritas en el ámbito regional. De este modo, se superaron las 60000 personas, entre estudiantes de diferentes niveles educativos (inicial, primaria, secundaria, técnica y superior), turistas locales, nacionales y extranjeros, que recibieron de algún modo mensajes de conservación y protección de los manatíes amazónicos, delfines de río y otras especies en peligro. Estas actividades además de la ciudad de Iquitos, se desarrollaron en comunidades campesinas y nativas, capitales distritales y provinciales del ámbito jurisdiccional de Loreto.

Así también, a través de ACOBIA-DWAZOO el FI-CREA, ganó el premio Voluntariado Ambiental 2010 del Ministerio del Ambiente y este año gana el premio Protagonistas del Cambio 2011, de la Universidad Peruana de Ciencias Aplicadas (UPC).

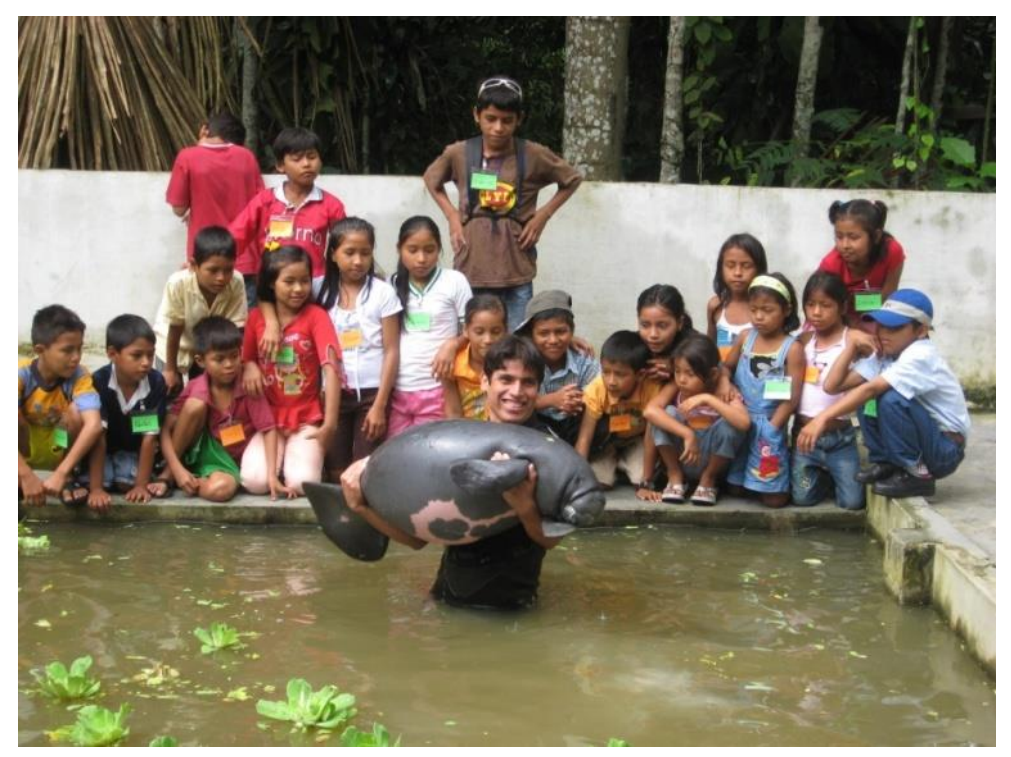

Figura 2: Manatí amazónico en rehabilitación en las instalaciones de la FI-CREA y exhibido como parte de actividades de educación ambiental.

\section{Investigación}

Se ha iniciado el desarrollo de investigaciones a través de tesis de pre grado y post grado, en temas tales como:

- Comportamiento de Manatí Amazónico (Trichechus inunguis) en cautiverio en las instalaciones del Centro de Rescate de ACOBIA-DWAZOO. Iquitos - Perú

- Determinación de los valores hematológicos y bioquímica sanguínea del manatí amazónico (Trichechus inunguis) mantenidos en cautiverio en el Centro de Rescate Amazónico en el departamento de Loreto.

- Diagnóstico preliminar sobre etnoconocimiento del Trichechus inunguis (Manatí Amazónico) en la cuenca del Pacaya Samiria, Región Loreto.

- Uso de hábitat en época seca de manatíes amazónicos (Trichechus inunguis) liberados en Cocha El Dorado - Reserva Nacional Pacaya Samiria.

- Áreas críticas de amenazas y conservación de Trichechus inunguis (Natterer 1883) en la Reserva Nacional Pacaya Samiria.

No obstante, la aplicación de tratamientos y manipulación de los ejemplares de manatí amazónico mantenidos en cautiverio en la FI-CREA, permitirá conocer aspectos sanitarios, de manejo, comportamiento, alimenticio, procesos de liberación y adaptación al medio natural, entre otros, así como la elaboración de protocolos e informes técnicos que permitirá al Estado peruano a través 
Cienc. amaz., Vol. 1, No. 2, 2011

del Ministerio de la Producción y del Gobierno Regional de Loreto, ordenar, normar, proteger y conservar a esta especie.

\section{CONCLUSIONES}

- En términos generales la implementación de la Fundación Iquitos - Centro de Rescate Amazónico ha permitido iniciar el proceso de conservación del manatí amazónico en el Perú, aunque aún con instalaciones provisionales, este Centro ofrece oportunidades de supervivencia para aquellos ejemplares que son recuperados, rescatados o decomisados por las autoridades competentes.

- La educación ambiental, es y será una herramienta importante y fundamental para que los esfuerzos de conservación tengan éxito, sobre todo en aquellas zonas donde aún perdura la caza y comercialización ilegal de esta especie.

- Sin embargo, es de suma prioridad la implementación de un Plan de Acción en torno a delfines de río y manatí en la Amazonía peruana, que sea reconocida, validada y aprobada por el Ministerio de la Producción, para su aplicación principalmente en los departamentos de Loreto, Ucayali, San Martín y Madre de Dios. Esta iniciativa debe ser compartida con experiencias de otros países tales como Colombia, Ecuador y Brasil.

\section{AGRADECIMIENTOS}

Al Dallas World Aquarium, por su apoyo incondicional en la titánica tarea de conservación del manatí amazónico en el Perú.

A la Dirección Regional de la Producción del Gobierno Regional de Loreto, cuyo plantel de profesionales contribuye permanentemente en la protección y conservación del manatí amazónico y de los delfines de río en el departamento de Loreto.

Al Instituto de Investigaciones de la Amazonía Peruana, especialmente al equipo de investigadores del AQUAREC, quienes facilitan que el FI-CREA se consolide como ente de referencia internacional del manatí amazónico. 


\section{REFERENCIAS BIBLIOGRÁFICAS}

CAICEDO-HERRERA, D., TRUJILLO, F., RODRÍGUEZ, C.L. and RIVERA, M. (Editors), 2005. Programa Nacional de Manejo y Conservación de Manatíes en Colombia. Ministerio del Medio Ambiente, Vivienda y Desarrollo Territorial. Fundación Omacha., Bogotá, 176 pp.

DA SILVA, V. M. 2009. Conservação y manejo do peixe-boi da Amazônia (Trichechus inunguis) em cativeiro. LMA-INPA.

PEREA-SICCHAR, C. M., M. ESPINOZA-AZAN, L. J. VELÁSQUEZ-VARELA, D. L. RICHARDSON and A. A. MIGNUCCI-GIANNONI. 2009. Status and conservation efforts of Amazonian manatees (Trichechus inunguis) in Perú. In: ABSTRACTS 18TH BIENNIAL CONFERENCE ON THE BIOLOGY OF MARINE MAMMALS. Québec - Canada. 198 p.

REEVES, R. R; E. LEATHERWOOD; T. A. JEFFERSON; B. E. CURRY and T. HENNINGSEN 1996. Amazonia manatees, Trichechus inunguis, in Peru: distribution, exploitation and conservation status. Interciencia, Vol. 21 (6).

VELÁSQUEZ-VARELA L. J., C. M. PEREA-SICCHAR, M. ESPINOZA-AZAN, N. SÁNCHEZ-PEREA, J. SÁNCHEZ-BABILONIA, R. LÓPEZ-PINHO, L. SINGLER, D. MURPHY, D. L. RICHARDSON and A. A. MIGNUCCI-GIANNONI. 2009. Rescue and rehabilitation of orphaned Amazonian manatee (Trichechus inunguis) in Peru. In: ABSTRACTS 18TH BIENNIAL CONFERENCE ON THE BIOLOGY OF MARINE MAMMALS. Québec - Canada. 266-267 p.

Recibido: 10 agosto 2011 / Aceptado: 20 octubre 2011 